\title{
Empty electron states in cobalt-intercalated graphene
}

Cite as: J. Chem. Phys. 153, 214703 (2020); https://doi.org/10.1063/5.0021814

Submitted: 14 July 2020 . Accepted: 06 November 2020 . Published Online: 01 December 2020

(iD) Alberto Calloni, (D) Gianlorenzo Bussetti, Giulia Avvisati, Madan S. Jagadeesh, Daniela Pacilè, (D) Andrea Ferretti, (i) Daniele Varsano, (D) Claudia Cardoso, Lamberto Duò, (D) Franco Ciccacci, and (D) Maria Grazia Betti

\section{ARTICLES YOU MAY BE INTERESTED IN}

First-principles description of electrocatalytic characteristics of graphene-like materials The Journal of Chemical Physics 153, 214704 (2020); https://doi.org/10.1063/5.0031106

Reflections on electron transfer theory

The Journal of Chemical Physics 153, 210401 (2020); https://doi.org/10.1063/5.0035434

Surprisal of a quantum state: Dynamics, compact representation, and coherence effects The Journal of Chemical Physics 153, 214105 (2020); https://doi.org/10.1063/5.0030272

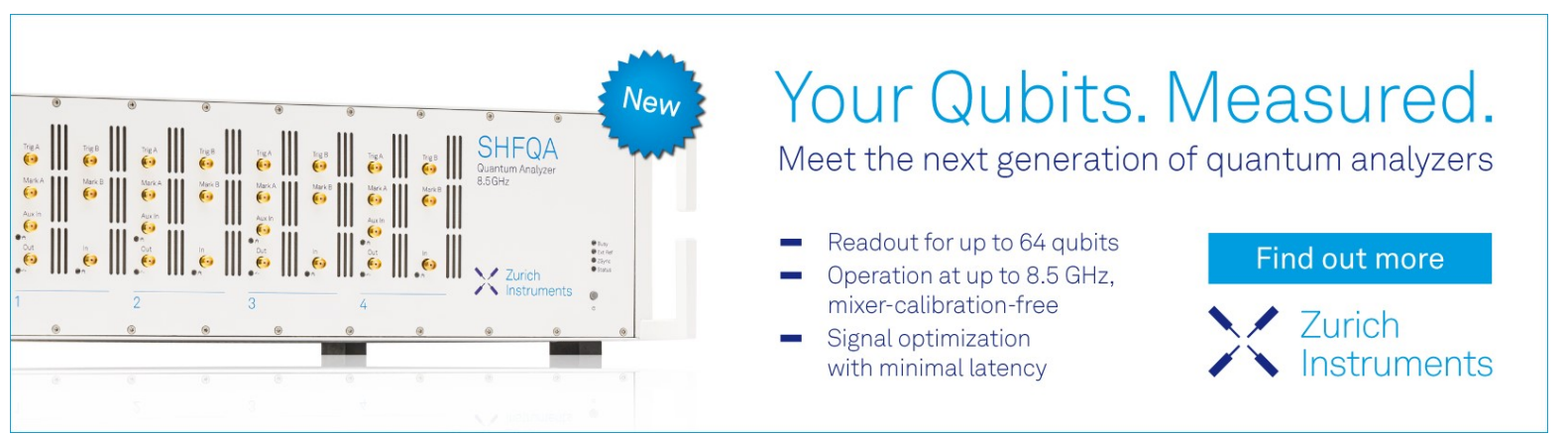




\title{
Empty electron states in cobalt-intercalated graphene
}

\author{
Cite as: J. Chem. Phys. 153, 214703 (2020); doi: 10.1063/5.0021814 \\ Submitted: 14 July 2020 - Accepted: 6 November 2020 • \\ Published Online: 1 December 2020
}

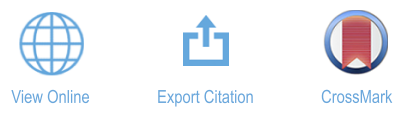

\begin{abstract}
Alberto Calloni, ${ }^{1, a)}$ (D) Gianlorenzo Bussetti, 'D Giulia Avvisati, ${ }^{2}$ Madan S. Jagadeesh, ${ }^{1}$ Daniela Pacilè,, Andrea Ferretti, ${ }^{4}$ (D) Daniele Varsano, ${ }^{4}$ (D) Claudia Cardoso, ${ }^{4}$ (D) Lamberto Duò, ${ }^{7}$ Franco Ciccacci, and Maria Grazia Betti ${ }^{2}$
\end{abstract}

\author{
AFFILIATIONS \\ ${ }^{1}$ Dipartimento di Fisica, Politecnico di Milano, piazza Leonardo da Vinci 32, I-20133 Milano, Italy \\ ²Dipartimento di Fisica, Università di Roma "La Sapienza", I-00185 Roma, Italy \\ ${ }^{3}$ Dipartimento di Fisica, Università della Calabria, I-87036 Arcavacata di Rende (Cs), Italy \\ ${ }^{4}$ Centro S3, CNR-Istituto Nanoscienze, I-41125 Modena, Italy
}

${ }^{a)}$ Author to whom correspondence should be addressed: alberto.calloni@polimi.it

\begin{abstract}
The dispersion of the electronic states of epitaxial graphene ( $\mathrm{Gr}$ ) depends significantly on the strength of the bonding with the underlying substrate. We report on empty electron states in cobalt-intercalated Gr grown on $\operatorname{Ir}(111)$, studied by angle-resolved inverse photoemission spectroscopy and $\mathrm{x}$-ray absorption spectroscopy, complemented with density functional theory calculations. The weakly bonded Gr on Ir preserves the peculiar spectroscopic features of the Gr band structure, and the empty spectral densities are almost unperturbed. Upon intercalation of a Co layer, the electronic response of the interface changes, with an intermixing of the Gr $\pi^{*}$ bands and Co $d$ states, which breaks the symmetry of $\pi / \sigma$ states, and a downshift of the upper part of the Gr Dirac cone. Similarly, the image potential of $\operatorname{Ir}(111)$ is unaltered by the Gr layer, while a downward shift is induced upon Co intercalation, as unveiled by the image state energy dispersion mapped in a large region of the surface Brillouin zone.
\end{abstract}

Published under license by AIP Publishing. https://doi.org/10.1063/5.0021814

\section{INTRODUCTION}

The intercalation of metal layers under graphene (Gr) has been the subject of keen interest in the past decade for fundamental and applied physics, in particular due to the possibility of creating a magnetic system with tunable properties. ${ }^{1-6}$ The main outcomes of the metal intercalation are the modulation of the Gr electronic structure near the Dirac cone, ${ }^{7-9}$ the evolution of the interfacial moiré pattern and surface corrugation, ${ }^{10-14}$ and the effects of $\mathrm{Gr}$ on protecting the vulnerable surface properties of the underneath substrate. In fact, thanks to its chemical stability, Gr protects highly reactive surfaces and stabilizes magnetic thin films against oxidation.

The electronic properties of epitaxial Gr significantly depend on the supporting substrate and on the interface. For instance, Gr grown on $\operatorname{Ir}(111)$ presents a rippled moiré superstructure, indicating a weak interaction with the metallic substrate and preserving the peculiar spectroscopic features of the Gr band structure, such as the Dirac cone. ${ }^{15,16}$ Conversely, the growth of $\mathrm{Gr}$ onto transition metal (TM) surfaces, such as $\mathrm{Ni}(111)$ or $\mathrm{Co}(0001)$, of interest for their ferromagnetic coupling, generally results in a better lattice matching at the expense of a perturbed band structure, ${ }^{17}$ as shown by angle-resolved photoemission spectroscopy (AR-PES). ${ }^{18,1}$

While the precise evolution of the Gr-filled electronic states and of the Dirac cone has been determined for a large class of intercalated $\mathrm{Gr}$ systems, ${ }^{20-22}$ the response of the empty states has been much less investigated. Recently, angle-resolved two photon-photoemission (AR-2PPE) ${ }^{23,24}$ and scanning tunneling spectroscopy ${ }^{25}$ have unveiled processes involving image-potential states (ISs) in metal surfaces covered by Gr. ISs are observed within the surface-projected bulk bandgap and are related to electrons 
trapped by the attractive image-charge potential just outside the surface. The response of ISs to the composite dielectric/metal systems involving $\mathrm{Gr}$ is itself of fundamental physical interest. Theoretically, a dual Rydberg-like series of even and odd symmetry image-potential states is expected in a single freestanding sheet of $\mathrm{Gr}^{2}$

The underlying metal substrate can break the mirror symmetry of the $\mathrm{Gr}$ layer as reported for $\mathrm{Gr}$ on $\mathrm{Ru}(0001)$ and $\mathrm{Gr}$ on $\mathrm{Pt}(111)$, while scanning tunneling spectroscopy measurements ${ }^{25}$ unveil two series of ISs in $\mathrm{Gr}$ single and bilayer grown on SiC. Image-potential states are very sensitive to any change of shape or/and environment of the Gr sheet. The topography, the interlayer and impurity interactions, the intercalated layers, and the evolution of the metallic surface states upon intercalation can all strongly affect the IS energy and dispersion. ${ }^{23,24}$ In this respect, angle-resolved inverse photoemission spectroscopy (AR-IPES) is an ideal probe to map empty states, and ISs in particular, especially when looking at a wide region of the surface Brillouin zone.

In this paper, we report on the direct band structure mapping of empty states for cobalt-intercalated Gr on $\operatorname{Ir}(111)$ by means of AR-IPES and Near Edge X-ray Absorption Fine Structure (NEXAFS). Noticeably, image-potential states are explicitly addressed by AR-IPES data. First principles simulations based on density functional theory (DFT) were also performed to calculate the electronic structure (DOS and bands) and simulate NEXAFS for Gr/Ir(111) and $\mathrm{Gr} / 1 \mathrm{ML}-\mathrm{Co} / \mathrm{Ir}(111)$. At variance with AR-2PPE, AR-IPES can explore uncharted regions of the energy space $E(\mathbf{k})$, allowing for a direct comparison of the evolution of the empty states of $\operatorname{Ir}(111)$, a single layer of $\mathrm{Gr}$ on $\operatorname{Ir}(111)$, and the cobalt layer sandwiched between $\mathrm{Gr}$ and $\operatorname{Ir}(111)$. Carbon-projected band dispersion for freestanding $\mathrm{Gr}$ and $\mathrm{Gr} / \mathrm{Co}(0001)$ systems is also analyzed and used to discuss the AR-IPES data.

This paper is organized as follows. In Sec. II A, we describe the experimental methods, including growth, characterization of the samples, NEXAFS, and AR-IPES setup, while in Sec. II B, we report the details of the first principles computational approach. Next, in Sec. III A, we discuss experimental and theoretical results, including NEXAFS data, concerning the characterization of the samples before and after cobalt intercalation. Finally, empty states, sampled using AR-IPES, are reported and discussed in Sec. III B, together with band structures computed at the Kohn-Sham DFT level.

\section{METHODS}

\section{A. Experimental details}

Sample preparation and AR-IPES measurements took place in an ultra-high vacuum system working at a base pressure in the $1 \times 10^{-10}$ mbar range. ${ }^{28}$ The $\operatorname{Ir}(111)$ substrate was cleaned by sputtering and annealing cycles (up to $1470 \mathrm{~K}$ ). The substrate was then exposed to oxygen at a pressure of $1.3 \times 10^{-7} \mathrm{mbar}$ (overall exposure is $8 \mathrm{~L}$, considering $1 \mathrm{~L}=1.33 \times 10^{-6} \mathrm{mbar} \times \mathrm{s}$ ) with the surface kept at $1170 \mathrm{~K}$ in order to segregate and desorb carbon. Subsequent flashes at $1470 \mathrm{~K}$ removed adsorbed oxygen. ${ }^{29}$ The quality of the samples and the consistency with literature data of the entire procedure are ensured by low energy electron diffraction (LEED) and photoemission (see Refs. 29 and 30 for more details). The Gr layer was prepared by repeatedly exposing the $\operatorname{Ir}(111)$ surface up to $45 \mathrm{~L}$ of ethylene $\left(\mathrm{C}_{2} \mathrm{H}_{4}\right)$ and annealing at $1300 \mathrm{~K}$. The quality of the Gr layer was then assessed by $\mathrm{x}$-ray photoemission, considering the $\operatorname{Ir} 4 f$ to $\mathrm{C} 1 s$ core level intensity ratio, ${ }^{31}$ and the sharpness of the moiré pattern with LEED.

Cobalt was deposited on $\mathrm{Gr} / \operatorname{Ir}(111)$ at room temperature (RT) by molecular beam epitaxy, using a homemade e-beam sublimation cell loaded with a high purity $(6 \mathrm{~N})$ Co rod. The intercalation of Co is achieved by annealing at about $670 \mathrm{~K}$ (i.e., well below the temperature threshold for Co-Ir interface alloying ${ }^{32,33}$ ). The nominal Co thickness was $2.5 \AA$, estimated by means of a quartz microbalance, i.e., slightly in excess of the Co ML thickness, assumed to be close to the bulk $\operatorname{Ir}(111)$ interplanar spacing $\left(2.22 \AA^{34}\right)$ for a pseudomorphic-type of growth.

IPES measurements were performed by extracting electrons with laser light from a $\mathrm{GaAs}(100)$ photocathode prepared according to standard procedures, ${ }^{35,36}$ and detecting $9.6 \mathrm{eV}$ photons emitted in the inverse photoemission process by means of a bandpass detector. The system operates in the isochromatic mode, i.e., at fixed photon energy and by changing the energy of impinging electrons. ${ }^{37-39}$ The full width at half maximum energy (angular) resolution is about $0.7 \mathrm{eV}\left(3^{\circ}\right)$.

NEXAFS and photoemission measurements were performed at the SuperESCA beamline of the synchrotron radiation facility ELETTRA (Trieste, Italy). The $\mathrm{C} \mathrm{K}$ edge was acquired in the Auger yield mode, collecting the tail of the KLL Auger electrons, at a kinetic energy of about $260 \mathrm{eV}$, within an energy window of $12 \mathrm{eV}$ and with an overall energy resolution of $100 \mathrm{meV}$. Measurements were acquired with horizontal linearly polarized radiation and with the electric field vector either parallel or almost normal (about $70^{\circ}$ ) with respect to the surface plane, by rotating the sample position.

\section{B. Computational details}

DFT simulations were performed using the plane-wave and pseudopotential implementation provided by the QUANTUM ESPRESSO distribution $^{40,41}$ using the Local Density Approximation (LDA) exchange-correlation functional, according to the Perdew-Zunger parameterization. ${ }^{42}$ Consistent with our previous work, ${ }^{2} \mathrm{Gr} / \mathrm{Ir}(111)$ and $\mathrm{Gr} / 1 \mathrm{ML}-\mathrm{Co} / \operatorname{Ir}(111)$ were simulated including the complete moiré-induced periodicity by using a $9 \times 9$ supercell of $\operatorname{Ir}(111)$, corresponding to a $10 \times 10$ supercell of pristine Gr. For comparison, freestanding $\mathrm{Gr}$ and $1 \times 1 \mathrm{Gr} / \mathrm{Co}(0001)$ were also computed.

Since, for the case of a single layer of Co intercalated under graphene, Co assumes the same structure of $\operatorname{Ir}(111)$ [see LEED in Fig. 1(d)], the moiré superstructures of $\mathrm{Gr} / \mathrm{Ir}(111)$ and $\mathrm{Gr} / 1 \mathrm{ML}$ $\mathrm{Co} / \operatorname{Ir}(111)$ have the same periodicity. In practice, we have treated the Co layer with the same registry of one extra Ir layer, except for the interlayer distance, which has been relaxed. For both interfaces, we have considered the lattice parameters of Ir bulk relaxed at the LDA level using ultrasoft pseudopotentials (USPP), resulting in an Ir-Ir distance of $2.7048 \AA$ (corresponding to a hexagonal cell of 46.001 Bohr radii) for the moiré structure. Four metallic layers (3 Ir plus one Co layer or 4 Ir layers) were included in the calculations. A layer of $\mathrm{H}$ atoms was included at the bottom of the slab to make the two sides of the slab inequivalent. Atomic 


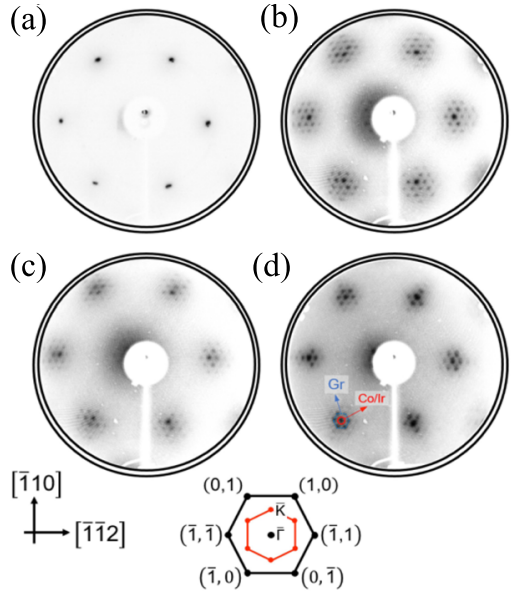

(e)
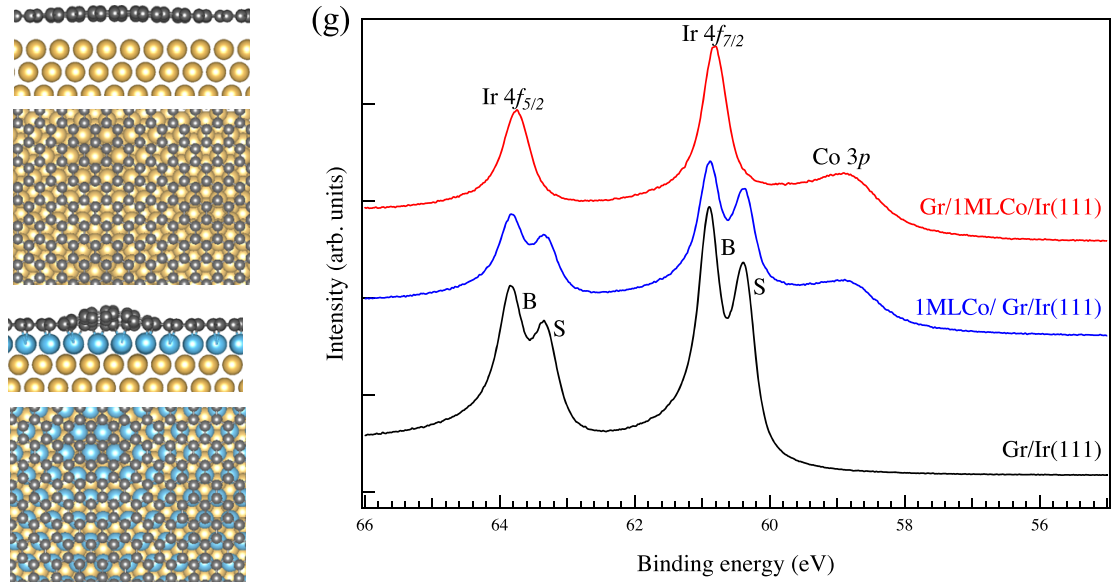

FIG. 1. Left panels: low energy electron diffraction (LEED) images taken at a beam energy of $140 \mathrm{eV}$ : (a) $\operatorname{lr}(111)$, (b) $\mathrm{Gr} / \mathrm{Ir}(111)$, (c) as deposited 1ML-Co/Gr/lr(111), and (d) after Co intercalation by annealing at $670 \mathrm{~K}$ [i.e., Gr/1ML-Co/lr(111)]. At the bottom: sketch of the diffraction pattern of the $\operatorname{lr}(111)$ surface. The surface Brillouin zone (SBZ) is shown in red. Middle panels: (e) side and top views of the corrugation and moiré pattern of $\mathrm{Gr} / \mathrm{lr}(111)$ and (f) of $\mathrm{Gr} / 1 \mathrm{ML}-\mathrm{Co} / \mathrm{lr}(111)$, as determined by DFT. Right panel: ( $\mathrm{g}$ ) Ir $4 f$ core level photoemission, taken at $h v=176 \mathrm{eV}$, of Gr/lr(111) (black curve), $1 \mathrm{ML}-\mathrm{Co} / \mathrm{Gr} / \mathrm{Ir}(111)$ (blue curve) and Gr/1ML-Co/lr(111) (red curve). All spectra show Ir $4 f_{5 / 2}$ and Ir $4 f_{7 / 2}$ core levels [with a bulk (B) and surface (S) component before Co intercalation], and Co $3 p$ core level (after Co deposition).

positions were then fully relaxed (except for the two bottom Ir layers and the $\mathrm{H}$ saturation layer) until ionic forces were smaller than $0.001 \mathrm{Ry} / \mathrm{bohr}$. All the self-consistent calculations were performed by using a $2 \times 2$ grid of k-points, norm-conserving pseudopotentials, and a kinetic energy cutoff of 75 Ry to represent Kohn-Sham wavefunctions.

NEXAFS spectra were simulated using the XSPECTRA code. ${ }^{43-45}$ The absorption from the core levels was simulated by considering a carbon pseudopotential including a half core-hole $(\mathrm{HCH}){ }^{46-49}$ One $\mathrm{HCH}$ atom per cell (i.e., every $200 \mathrm{C}$ atoms), substituting a regular $\mathrm{C}$ atom, was placed at different positions in order to sample valley, intermediate, and peak regions of the moiré superstructure, as well as the two sub-lattices of Gr. The final spectrum was obtained by averaging the spectra over 8 selected sampling points. More details on the averaging procedure are provided in the supplementary material.

The C-projected band structures for $\mathrm{Gr} / \mathrm{Co}(0001)$ in the $1 \times 1$ unit cell (shown in Fig. 6) have been computed for a slab including $\mathrm{Gr}, 10$ layers of $\mathrm{Co}$, and a saturating back layer of $\mathrm{H}$ atoms. The lattice parameter of the slab $(2.430 \AA)$ has been taken from a full relaxation of the Co hcp structure, done at the DFT-LDA level (see also the supplementary material for more details). Comparison with calculations done using 4,6 , and 8 Co layers is provided in the supplementary material.

\section{RESULTS AND DISCUSSION}

\section{A. Co intercalation and NEXAFS data}

The intercalation of a single layer of cobalt, sandwiched between the $\operatorname{Ir}(111)$ surface and Gr, results in a corrugated Gr layer with a moiré superstructure superimposed to the hexagonal lattice of Gr, as deduced from the LEED patterns reported in the left panels of Fig. 1. The Ir(111) LEED [Fig. 1(a)] shows bright hexagonal spots and after Gr growth [Fig. 1(b)], a larger hexagonal pattern surrounded by hexagonal satellites (up to third order diffraction features), consistent with the smaller Gr lattice parameter, and a moiré superstructure caused by the lattice mismatch between $\mathrm{Gr}$ and $\mathrm{Ir}^{50}$ After Co deposition [Fig. 1(c)], the surface shows an unaltered pattern, only slightly attenuated after the Co addition, while the number of satellite spots is reduced when the Co layer is intercalated underneath Gr [Fig. 1(d)]. These differences are likely correlated with the changes in the Gr morphology in the presence of the Co intercalation.

The Co-C interaction in the moiré superstructure depends on the $\mathrm{C}$ site, with the result of increasing the corrugation of the Gr layer. The interaction with $\mathrm{Gr}$ is stronger in top-hollow and bridge sites, while the van der Waals-like interaction is reported for other sites (showing fcc/hcp stacking), ${ }^{19}$ as confirmed by DFT structural models. ${ }^{2,31,51}$ The increase of the Gr corrugation upon Co intercalation and the structural details of the moiré structures, as computed in our DFT calculations, are illustrated in Figs. 1(e) and $1(\mathrm{f})$.

The graphene layer, almost unperturbed by the $\operatorname{Ir}(111)$ underlying surface, is highly perturbed upon Co intercalation, and a strong $\mathrm{C}-\mathrm{Co}$ interaction is observed. This can be clearly unveiled by photoemission from the $\operatorname{Ir} 4 f_{5 / 2}$ and Ir $4 f_{7 / 2}$ core levels, both with components originating from bulk (B) and surface (S), as shown in Fig. $1(\mathrm{~g})$. The surface components, at about $0.5 \mathrm{eV}$ lower binding energy, due to emission from the topmost atomic layer of $\operatorname{Ir}(111)$, are unaffected by the formation of the Gr layer (black curve), corroborating the formation of a quasi-freestanding electronic structure of Gr, and they are essentially unchanged after the deposition of Co on Gr (blue curve, a mere attenuation of both surface and volume contributions is seen as due to the Co overlayer). Coming to Co intercalation under the graphene layer (red curve), the 
Co $3 p$ levels are only slightly attenuated in intensity with respect to the non-intercalated case (blue curve). While a $\mathrm{Co}_{x} \operatorname{Ir}_{1-x}$ interface alloying has been recently reported at annealing temperatures higher than $900 \mathrm{~K},{ }^{33}$ here, the intensity ratio between Co $3 p$ and Ir $4 f$ states is almost unchanged, showing that at the intercalation temperature (well below $900 \mathrm{~K}$ ), the bulk solubility of Co atoms must be negligible.

On the other side, the intercalation of $\mathrm{Co}$ at $670 \mathrm{~K}$ induces a sudden quenching of the Ir surface components [Fig. 1(g), red curve] and strongly influences the photoemission from the overlying Gr membrane, as exhaustively discussed in previous papers. ${ }^{19,31}$ Moreover, cobalt deposited on top of the Gr layer does not influence the $\mathrm{C} 1 \mathrm{~s}$ line shape, as shown in the spectra reported in the supplementary material. After intercalation, the C $1 s$ core level photoemission presents a multi-component line shape with two main features, at $284.42 \mathrm{eV}$ and $284.92 \mathrm{eV} .{ }^{19,31}$ These are assigned to the $\mathrm{C}$ atoms in the Gr layer weakly and strongly bound to the intercalated Co layer, respectively. Such experimental evidence and the disappearance of the Ir surface components prove a homogeneous Co intercalation, featuring a Co-Gr interaction upon intercalation with a redistribution of the charge density at the $\mathrm{Gr} / 1 \mathrm{ML}-\mathrm{Co} / \mathrm{Ir}(111)$ interface, in turn preventing any Co-Ir alloying.
A further signature of the interaction of corrugated $\mathrm{Gr}$ with the underlying Co layer can be highlighted by the $\mathrm{C} \mathrm{K}$ absorption spectra (NEXAFS) for $\mathrm{Gr} / \mathrm{Ir}(111)$ and $\mathrm{Gr} / 1 \mathrm{ML}-\mathrm{Co} / \mathrm{Ir}(111)$ reported in panels [(a) and (b)] of Fig. 2. The electric field oriented almost normal $\left(70^{\circ}\right.$ with respect to the surface plane) allows one to enhance the signal from the $\pi^{*}$ states, even though this setup does not completely quench the $\sigma^{*}$ state contribution. Concerning $\mathrm{Gr} / \mathrm{Ir}(111)$, the main peak at $285.5 \mathrm{eV}$ is associated with the $\pi^{*}$ conduction states. Moreover, a shoulder located at about $284.2 \mathrm{eV}$ can be seen in Fig. 2(a). This feature observed for single layer Gr on metals, graphene flakes ${ }^{52,53}$ and nano-graphite grains, ${ }^{54}$ was attributed to edge-derived electronic states. However, in the present case, the highly ordered corrugated $\mathrm{Gr}$ structure with large single domains cannot justify a contribution from edge defects to the pre-edge feature. On the other hand, a small $p$-doping observed for $\mathrm{Gr} / \mathrm{Ir}(111)^{55}$ can induce a tiny density of empty states below the Dirac cone apex available for the excited core electrons. This is supported by our DFT simulations where the comparison between the DOS computed for freestanding $\mathrm{Gr}$ and $\mathrm{Gr}$ on Ir reveals a small $p$-doping corresponding to an excess charge of about $1.1 \times 10^{-3}$ electrons per $\mathrm{C}$ atom (DOS plots and more details in Fig. S4 of the supplementary material). The higher energy features are due to transitions toward higher-lying states resonant with contributions coming from the
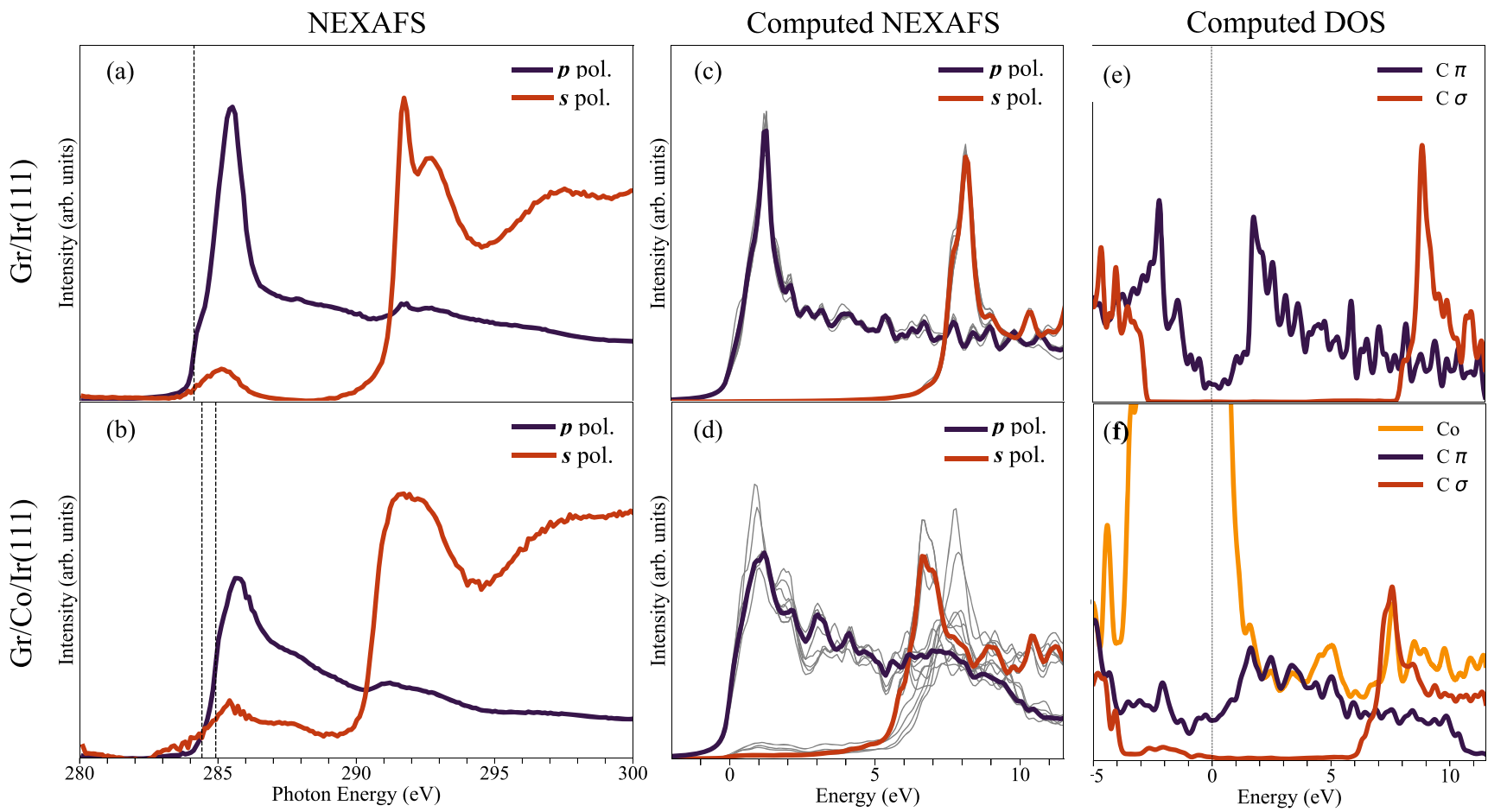

FIG. 2. Measured NEXAFS [(a) and (b)], computed NEXAFS [(c) and (d)], and DOS data [(e) and (f)] for Gr/lr(111) (upper panels) and Gr/1ML-Co/lr(111) (lower panels) respectively. Purple and red lines correspond to $C 1 s$ levels excited by out-of-plane and in-plane polarized photons. Vertical dashed lines correspond to the Fermi level reference, as deduced by the energy position of the $\mathrm{C} 1 \mathrm{~s}$ core levels $(284.13 \mathrm{eV}$ for $\mathrm{Gr} / \mathrm{lr} ; 284.92 \mathrm{eV}$ and $284.42 \mathrm{for} \mathrm{Gr} / \mathrm{Co} / \mathrm{lr})$. Central panels: simulated NEXAFS spectra averaged over different positions of the half core-hole (see the supplementary material). The gray lines represent the spectra computed for different core-holes and give an idea of the initial-state induced broadening of the spectra. Right panels: computed DOS projected on Co and $\mathrm{Gr} \sigma\left(\mathrm{C} s+p_{x}+p_{y}\right)$ and $\pi\left(\mathrm{C} p_{z}\right)$ orbitals for the same systems. The zero of the energy scale corresponds to the Fermi level. 
$\sigma^{*}$ conduction states, thus more visible in the $s$ configuration (red curve).

The measured $\mathrm{C} \mathrm{K}$ absorption edge of $\mathrm{Gr} / 1 \mathrm{ML}-\mathrm{Co} / \mathrm{Ir}(111)$ is reported in Fig. 2(b). Three main signatures diversify the NEXAFS spectra before and after the Co intercalation: (i) the disappearance of the pre-edge feature due to the small $p$-doping observed for $\mathrm{Gr} / \operatorname{Ir}(111)$, (ii) a broadening of the spectral features related to the $\pi^{*}$ and $\sigma^{*}$ transitions, and (iii) an energy shift of the absorption onset larger for the $\sigma^{*}$ transitions than for the $\pi^{*}$ ones.

The quenching of the pre-edge can be ascribed to the removal of $p$-doping due to a shift of the Dirac cone, as described in the computed band reported in Fig. 6 (Sec. III B). As far as it concerns the $\pi^{*}$ transitions, the main peak, centered at $285.7 \mathrm{eV}$, appears weaker and broader. Moreover, in the energy region above the onset, between $287 \mathrm{eV}$ and $290 \mathrm{eV}$, a wide shoulder appears in both $p$ and $s$ configurations, as observed also for Gr grown on highly interacting substrates like $\mathrm{Gr} / \mathrm{Ru}(0001), \mathrm{Gr} / \mathrm{Rh}(111)$, or $\mathrm{Gr} / \mathrm{Ni}(111) .{ }^{56}$ The origin of the smearing of these features can be due to both initial state effects and hybridization of the out-of-plane C- $\pi$ and Co- $d$ final states. The initial state effect is due to the spatially modulated adsorption potential of $\mathrm{C}$ upon Co-intercalation, which stems from the moiré structure of $\mathrm{Gr} / \mathrm{Ir}(111)$, reflected in different binding energies of the $\mathrm{C} 1 s \mathrm{Gr}$ core levels. After intercalation, the $\mathrm{C} 1 s$ core level photoemission presents a multi-component line shape with two main features, at $284.42 \mathrm{eV}$ and $284.92 \mathrm{eV},{ }^{19,31}$ assigned to $\mathrm{C}$ atoms in the $\mathrm{Gr}$ layer weakly and strongly bound to the intercalated Co layer, respectively, as reported in the supplementary material.

The presence of these features induces a convolution of a multiedge onset; however, they cannot fully explain the large broadening of the spectral density in the energy range of $285 \mathrm{eV}-290 \mathrm{eV}$, where the contribution from the hybridized $\mathrm{Co}-\mathrm{C}$ states dominates. As supported by the DFT calculations [see below and Fig. 2(f)], a contribution to the $\pi^{*}$ transition spectral density can be certainly ascribed to the hybridization of C- $\pi$ and Co states induced by the intercalation. Such contribution is further evidenced by changing the light polarization from $p$ to $s$. In fact, in-plane $\sigma^{*}$ states present an onset at about $291 \mathrm{eV}$ for $\mathrm{Gr} / \mathrm{Ir}(111)$ with two well-defined structures, while for Gr/1ML-Co/Ir(111), the absorption from the $\sigma^{*}$ states is observed also at lower photon energies $(285 \mathrm{eV}-290 \mathrm{eV})$, with slightly smeared structures. This is consistent with a broken symmetry of the $\pi^{*}$-conduction states as mixed with the Co $d$ states, resulting in a non-negligible signal with $s$-polarization.

In order to interpret the experimental results discussed above, we have simulated the NEXAFS spectra for the in-plane and outof-plane polarizations using DFT and including core-hole effects (see Sec. II B). The results are reported in Figs. 2(c) and 2(d) for $\mathrm{Gr} / \mathrm{Ir}(111)$ and $\mathrm{Gr} / 1 \mathrm{ML}-\mathrm{Co} / \operatorname{Ir}(111)$, respectively. In both figures, we show the spectra computed considering the half core-hole located on different carbon atoms, sampling moiré valleys, hills, and intermediate regions (gray lines) and the final spectra obtained by performing a weighted average (blue and red lines) that takes into account the $\mathrm{C}$-metal distance, as detailed in the supplementary material. The comparison of the averaged spectra clearly shows a broadening of the peaks for $\mathrm{Gr} / 1 \mathrm{ML}-\mathrm{Co} / \mathrm{Ir}(111)$ when compared with $\mathrm{Gr} / \mathrm{Ir}(111)$, in agreement with experiments.
In order to further clarify the differences seen in the NEXAFS spectra after Co intercalation, we report in Figs. 2(e) and 2(f) the computed projected density of states (where no core-hole is considered) of the two systems, focusing on the Gr $\pi^{*}$ - and $\sigma^{*}$ projections. A less structured and more broadened DOS is clearly evident for the case of $\mathrm{Gr}$ in $\mathrm{Gr} / 1 \mathrm{ML}-\mathrm{Co} / \mathrm{Ir}(111)$, confirming the idea of a contribution from the change of the final-state eigenpairs to the experimentally observed broadening of the NEXAFS spectrum. The $\sigma^{*}$ states located at $8.9 \mathrm{eV}$ for $\mathrm{Gr} / \mathrm{Ir}(111)$ are also shifted toward lower energies, at $7.6 \mathrm{eV}$, for $\mathrm{Gr} / 1 \mathrm{ML}-\mathrm{Co} / \operatorname{Ir}(111)$. Such a shift allows for the superposition of the Gr $\sigma^{*}$ and Co peaks and is in agreement with the downshift of the $\sigma^{*}$ peaks seen for $\mathrm{Gr} / 1 \mathrm{ML}-\mathrm{Co} / \mathrm{Ir}(111)$, in the measured and computed NEXAFS spectra.

By comparing the NEXAFS spectra obtained with the half core-hole located on different carbon atoms, it is evident that, in the case of $\mathrm{Gr} / \operatorname{Ir}(111)$, all the spectra are nearly superimposed. In contrast, for $\mathrm{Gr} / 1 \mathrm{ML}-\mathrm{Co} / \operatorname{Ir}(111)$, the position of the maxima of the different spectra spans a larger range of energies. This initial state effect is clearly one of the contributions to the smearing of the final averaged spectra. On the other hand, a close look at the same individual contributions also shows that the Gr/1ML$\mathrm{Co} / \operatorname{Ir}(111)$ spectra present broader features than $\mathrm{Gr} / \operatorname{Ir}(111)$, which can be ascribed to the hybridized Co-C states (changes in the final state wavefunctions and eigenvalues). We can then conclude that both mechanisms (changes in the initial- and final-state eigenpairs) contribute to the observed broadening of the NEXAFS spectra.

Besides the broadening of the spectra, a shift in the energy position of the $\sigma^{*}$ and $\pi^{*}$ main peaks of Gr/1ML-Co/Ir(111) with respect to $\mathrm{Gr} / \operatorname{Ir}(111)$ is observed. In agreement with experiments, where a shift of about $0.8 \mathrm{~V}$ is measured, the main $\sigma^{*}$ peak is found in calculations $1.1 \mathrm{eV}$ lower in energy for Gr/1ML-Co/Ir(111) than for $\mathrm{Gr} / \mathrm{Ir}(111)$. This means that the weaker the $\mathrm{Gr}$-metal interaction [Gr/Ir(111) compared to Gr/1ML-Co/Ir(111), but also C atoms further away from the surface in the moiré structure of $\mathrm{Gr} / 1 \mathrm{ML}$ $\mathrm{Co} / \operatorname{Ir}(111)]$, the higher the energy of the main $\sigma^{*}$ peak in the NEXAFS spectra. A downshift in the energy of the electronic $\sigma^{*}$ bands calculated for Gr/Co with respect the Gr layer is reported in Fig. 6 (Sec. III B), confirming the observed shift of the onset of the $\sigma^{*}$ transition in the $\mathrm{C} \mathrm{K}$ edge and the influence of the Co layer under graphene even on the empty states far from the Fermi level.

The detailed comparison between the $\mathrm{C} \mathrm{K}$ edge and the simulated NEXAFS spectra and the projected density of empty states clearly reproduce both the broadening and the energy shift of the $\mathrm{C}$ states, and the superposition of these states with Co states, signatures of the hybridization between $\mathrm{Gr}$ and Co orbitals, resulting also in the spin polarization of the Gr states. It is interesting to notice that these features are already present in the computed DOS of $\mathrm{Gr} / \mathrm{Co}(0001)$, in which the Gr corrugation due to the moiré pattern is not included. In fact, Fig. S4 of the supplementary material and Fig. 6 in Sec. III B, again corroborate the hybridization mechanism as responsible for the broadening observed in the NEXAFS spectra. This adds up to the structural effect due to the moiré structure, being, however, much smaller than the hybridization mechanism, as shown in the supplementary material. 


\section{B. Co intercalation: Angle-resolved inverse photoemission}

We will now address the empty electronic structure, paying special attention to image-potential states (ISs). Figure 3 displays the AR-IPES spectra acquired on clean $\operatorname{Ir}(111), \mathrm{Gr} / \operatorname{Ir}(111)$, and $\mathrm{Gr} / 1 \mathrm{ML}-\mathrm{Co} / \mathrm{Ir}(111)$. The angle-resolved measurements were performed by rotating the sample from normal incidence to $18^{\circ}$ along the $[\overline{1} \overline{1} 2]_{\text {Ir }}$ axis, i.e., along the $\bar{\Gamma}-\bar{K}$ direction of the surface Brillouin zone (SBZ), shown below the [(c) and (d)] panels of Fig. 1. The $\operatorname{Ir}(111)$ spectra [Fig. 3(a)] are characterized by two main features. The first one, slightly dispersive and located close to the Fermi level $\left(E_{\mathrm{F}}\right)$, is related to transitions toward Ir $5 d$ states. ${ }^{29}$ The second feature, labeled $I$, is detected at $5.1 \mathrm{eV}$. Considering a work function of $5.8 \mathrm{eV}$ for $\operatorname{Ir}(111),{ }^{57}$ this corresponds to a binding energy (BE) (with respect to the vacuum level) of $0.7 \mathrm{eV}$ for the $I$ state, which is well within the $\mathrm{BE}$ range reported for the $\mathrm{n}=1$ IS of several other TM (111) surfaces. ${ }^{58,59}$

AR-IPES spectra from $\mathrm{Gr} / \mathrm{Ir}(111)$ are reported in Fig. 3(b). They do not present any appreciable difference with respect to the clean $\operatorname{Ir}(111)$ surface, since Gr basically grows as an almost freestanding ordered sheet on $\operatorname{Ir}(111)$, without affecting the states localized at the surface. ${ }^{60}$ An important exception is peak $I^{\prime}$, which shifts toward lower energies, lying at $3.9 \mathrm{eV}$ in the normal incidence spectrum. A similar feature is reported for both HOPG and Gr, and it is attributed to an image potential state. ${ }^{12,61}$ Considering that epitaxial $\mathrm{Gr}$ decreases the work function of $\operatorname{Ir}(111)$ to $4.65 \mathrm{eV},{ }^{57}$ the $\mathrm{BE}$ of such a state is $0.8 \mathrm{eV}$, i.e., similar to the value found for $\operatorname{Ir}(111)$. Co deposition on top of the graphene layer leaves the ARIPES spectral density almost unperturbed, except for a decrease in the intensity of all the empty electronic states with respect to the Ir substrate.

Finally, AR-IPES spectra for Co-intercalated $\operatorname{Gr} / \operatorname{Ir}(111)$, reported in Fig. 3(c), show an attenuation of bulk features close to $E_{\mathrm{F}}$, while the peak corresponding to the IS labeled $I^{\prime \prime}$ is now

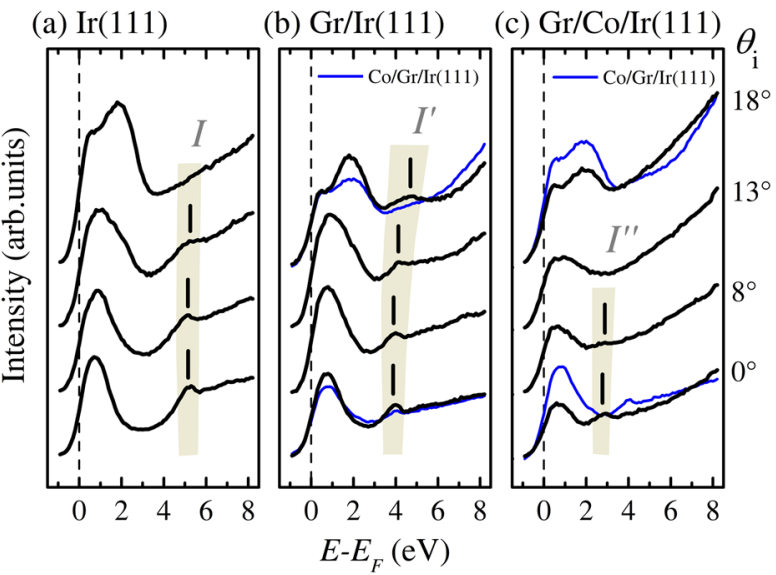

FIG. 3. AR-IPES spectra taken at various angles along the $\bar{\Gamma}-\bar{K}$ direction of: (a) Ir(111), (b) Gr/lr(111), and (c) Gr/1ML-Co/lr(111). Spectra related to as-deposited $1 \mathrm{ML}-\mathrm{Co} / \mathrm{Gr} / \mathrm{Ir}(111)$, acquired at selected angles, are drawn in panels (b) and (c) with thinner blue lines after an appropriate normalization. The spectra were acquired at room temperature. located at about $2.8 \mathrm{eV}$. The work function of $\mathrm{Gr} / 1 \mathrm{ML}-\mathrm{Co} / \mathrm{Ir}(111)$ is considerably reduced to a value around $3.3 \mathrm{eV},{ }^{62}$ thus giving an IS binding energy of about $0.5 \mathrm{eV}$ in this system. After the intercalation of the first Co layer, the surface component of the Ir $4 f$ surface states is quenched and the Rashba-Shockley state disappears, ${ }^{60}$ as observed in photoemission experiments. These $\operatorname{Ir}(111)$ surface hallmarks, preserved with the graphene $\operatorname{cover}^{60}$ and also when cobalt is deposited on top, are damped by the Co-Ir interaction if the Co layer is sandwiched between graphene and the Ir substrate, inducing a strong modification of the surface potential. The characteristics of the $I^{\prime \prime}$ state, therefore, account for the modifications in the surface potential due to the work function lowering ${ }^{62}$ and a different screening due to the presence of the metal layer on $\operatorname{Ir}(111)$.

The energy dispersion $E(\mathbf{k})$ along the $\bar{\Gamma}-\overline{\mathrm{K}}$ direction of the image-potential states $I, I^{\prime}$, and $I^{\prime \prime}$ is shown in Fig. 4, together with the experimental results of Ref. 23 measured by means of two-photon photoemission. The $I$ and $I^{\prime \prime}$ states of $\operatorname{Ir}(111)$ and Gr/1ML-Co/Ir(111) present a small dispersion, not allowing for a sensible evaluation of the their corresponding effective masses. This is instead possible for the $I^{\prime}$ state, visible on a larger $\mathbf{k}$ space region and showing a sizable dispersion. The parabolic fit of the energy dispersion, shown as a red line in Fig. 4, gives an effective mass of $1.1 \mathrm{me}_{\mathrm{e}}$. In addition, the analysis of the $I^{\prime}$ state on a wider $\mathbf{k}$-space region definitively confirms the attribution of this feature to an IS done in the previously reported $2 \mathrm{PPE}$ analysis. ${ }^{2}$

In order to explore a k-region closer to the $\overline{\mathrm{K}}$ point of the SBZ, where C-derived structures are expected, we extended the AR-IPES investigation to larger incidence angles for the $\mathrm{Gr} / \mathrm{Ir}(111)$ and the

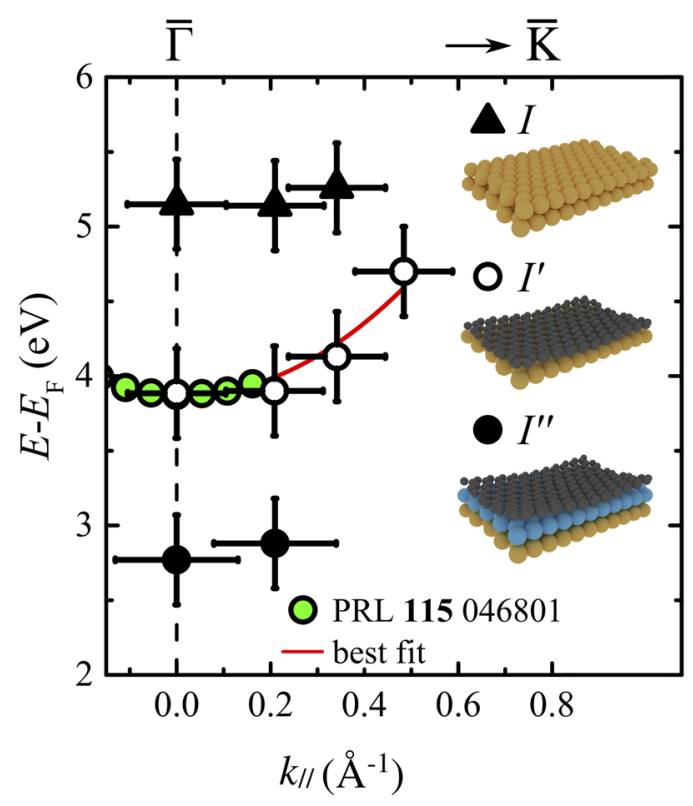

FIG. 4. Experimental AR-IPES data showing the momentum dispersion of states $I, I^{\prime}$, and $I^{\prime \prime}$. Experimental results from Ref. 23 are added for comparison. The red line represents the best fit of $I^{\prime}$ dispersion. 
Gr/1ML-Co/Ir(111) samples. Our results are presented in Figs. 5(a) and $5(\mathrm{~b})$ : close to $E_{\mathrm{F}}$, the spectra are dominated by the $\operatorname{Ir}(111)$ substrate contribution, as discussed in the supplementary material. We focus here on the most significant features present at higher energies, highlighted by a shaded area and labeled as $G^{\prime}$ and $G^{\prime \prime}$ for $\mathrm{Gr} / \mathrm{Ir}(111)$ and $\mathrm{Gr} / 1 \mathrm{ML}-\mathrm{Co} / \mathrm{Ir}(111)$, respectively. In the case of $\mathrm{Gr} / \mathrm{Ir}(111)$, the shape, intensity, and angular dispersion of $G^{\prime}$ are in good agreement with the AR-IPES results of highly oriented pyrolytic graphite $(\mathrm{HOPG})^{61}$ and of multilayer $\mathrm{Gr}$ grown on $6 \mathrm{H}-\mathrm{SiC}(0001)$, ${ }^{12}$ where $G^{\prime}$ is attributed to an electronic transition to the anti-bonding $\pi^{*}$ state. For increasing incidence angle, $G^{\prime}$ shifts toward lower energies. Its $E(\mathbf{k})$ dispersion is plotted in Fig. 6 (orange dots), where the experimental points show a linear dispersion whose extrapolation crosses $E_{\mathrm{F}}$ at the $\overline{\mathrm{K}}$ point of the $\mathrm{SBZ}$, as expected for the Dirac cone of Gr. Cobalt deposited on top of the graphene layer does not influence the topology of the Dirac cone, and the linearity is preserved, as deduced by the blue spectra reported in Fig. 5 for $1 \mathrm{ML}-\mathrm{Co} / \mathrm{Gr} / \operatorname{Ir}(111)$. Similar peaks related to transitions to the $\pi^{*}$ states of the Dirac cone are also found for Gr/1ML-Co/Ir(111), but shifted about $1 \mathrm{eV}$ toward lower energies. They are labeled as $G^{\prime \prime}$ in Fig. 5(b), and their dispersion is shown in Fig. 6 (light blue dots).

In order to better understand the $E(\mathbf{k})$ evolution of $\mathrm{Gr}$ empty states, we compare our AR-IPES data with the band structure computed within Kohn-Sham DFT. For a full theoretical simulation, it would be in principle necessary to compute the bands for a $10 \times 10$ Gr layer on top of the metal surface, and then to unfold the electronic states to the Gr primitive cell. ${ }^{64-66}$ However, we preferred to follow a simpler approach performing simulations for the $1 \times 1$ counterparts of $\mathrm{Gr} / \mathrm{Ir}(111)$ and $\mathrm{Gr} / 1 \mathrm{ML}-\mathrm{Co} / \mathrm{Ir}(111)$, namely, freestanding $\mathrm{Gr}$ and $\mathrm{Gr} / \mathrm{Co}(0001)$ treated in their primitive cells. The choice of freestanding graphene is justified by the weak $\mathrm{Gr} / \mathrm{Ir}$ interaction. The

(a) $\mathrm{Gr} / \mathrm{Ir}(111)$

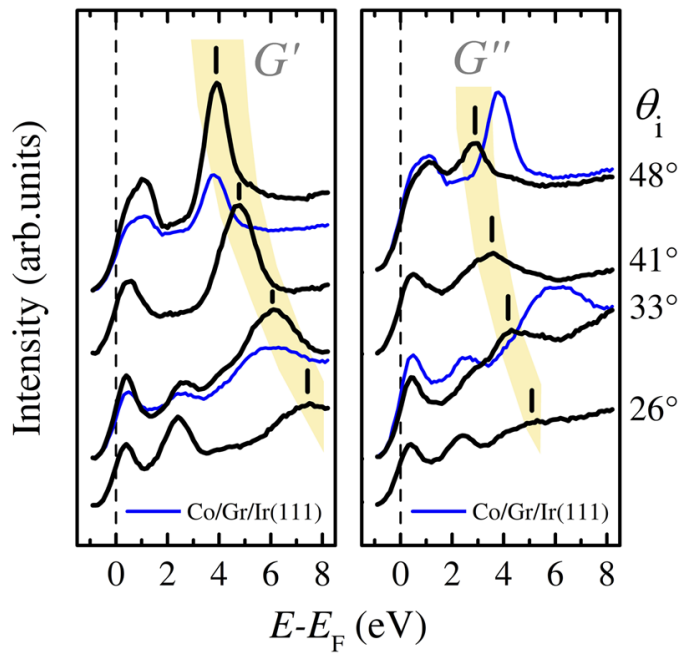

FIG. 5. AR-IPES spectra taken at various angles along the $\bar{\Gamma}-\bar{K}$ direction of: (a) Gr/lr(111) and (b) Gr/1ML-Co/lr(111). Spectra related to as-deposited 1ML$\mathrm{Co} / \mathrm{Gr} / \mathrm{Ir}(111)$, acquired at selected angles, are drawn with thinner blue lines after an appropriate normalization.

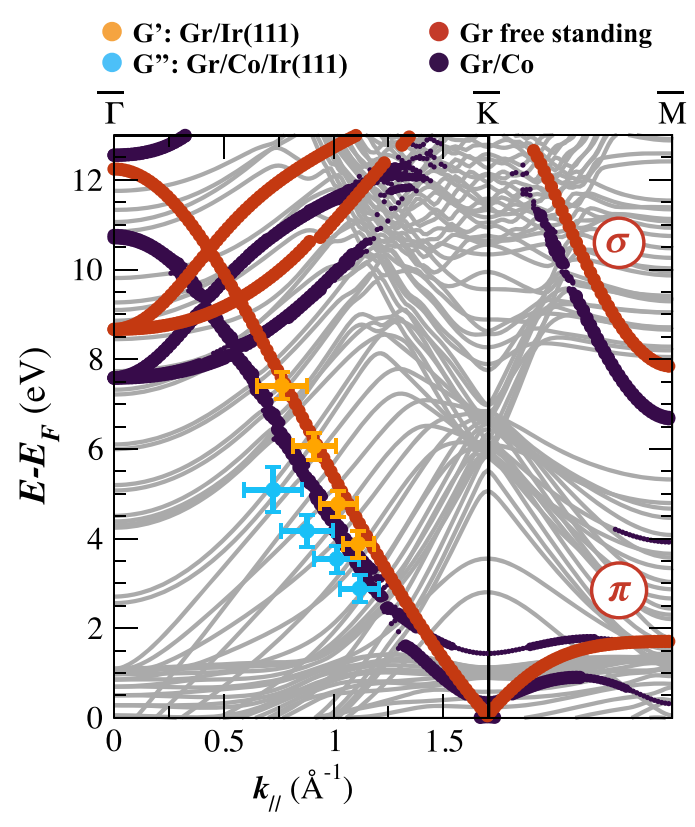

FIG. 6. AR-IPES data for Gr/Ir(111) ( $G^{\prime}$, orange dots) and Gr/1ML-Co/lr(111) $\left(G^{\prime \prime}\right.$, light blue dots) compared to the bands computed, respectively, for freestanding $\mathrm{Gr}$ (red dots) and flat $\mathrm{Gr} / \mathrm{Co}(0001)$ (blue dots), taken as models of the two measured systems as explained in the main text. The bands were computed in the $1 \times 1$ unit cell and projected on atomic orbitals (after a Lödwin orthogonalization is performed, as implemented in the QUANTUM ESPRESSO package ${ }^{40,41}$ ). In the plot, the size of the dots corresponds to the projection amplitude of the Kohn-Sham orbitals of the whole system onto $\mathrm{C}$ atomic orbitals (graphene). For simplicity, only four dot sizes are shown, corresponding to $5 \%, 15 \%, 30 \%$, and $50 \%$, respectively. Using the LDA lattice parameter of Co-bulk $(a=2.430 \AA), \bar{K}$ is located at $1.7238 \AA^{-1}$. This is compatible with the $\bar{K}$ point of the Gr/lr(111) SBZ, set at $1.70 \pm 0.02 \AA^{-1}$ from LEED and in agreement with Ref. 18.

$\mathrm{Co}(0001)$ surface, in contrast with Ir, has a lattice parameter similar to graphene, and therefore, the interface can be described by a $1 \times 1$ unit cell. On the other hand, the fact that the two components of the interface are commensurate results in a graphene sheet with no corrugation, contrary to what happens for Gr/1ML$\mathrm{Co} / \mathrm{Ir}(111)$. The validity of the above approximation is supported by the similarity of the electronic structure of the systems, as explained in detail in the supplementary material, and allows for a simpler analysis of the band dispersion. The computed results for the Gr $\pi^{*}$ bands are also reported in Fig. 6 as red and blue dots for $\mathrm{Gr}$ and $\mathrm{Gr} / \mathrm{Co}(0001)$, respectively. The size of the dots corresponds to the projection amplitude of the Kohn-Sham orbitals of the whole system onto atomic orbitals of graphene, and the labels, $\pi$ and $\sigma$, correspond to $\mathrm{C} p_{z}$ and $\mathrm{C}\left(s, p_{x}, p_{y}\right)$ orbital symmetries, respectively. In the figure, the dot scale ranges from $5 \%$ to $50 \%$, indicating a projection amplitude of at least the given value, justifying the apparent discontinuity of the bands. A plot including the $\mathrm{C}$ projected bands for a larger reciprocal space region is presented in Fig. S5 of the supplementary material. Notwithstanding the difference between the systems investigated, a good qualitative agreement is found between the experimental $G^{\prime}$ feature and the dispersion of the $\pi^{*}$ bands of 
Gr. Similar to freestanding Gr, the $\pi^{*}$ band starts at the $\bar{\Gamma}$ point at a large energy $(10.75 \mathrm{eV})$ and ends up close to $E_{\mathrm{F}}$ at the $\overline{\mathrm{K}}$ point, forming a Dirac cone. Good agreement is also found between the experimental behavior of the $G^{\prime \prime}$ feature and the theoretical modeling. In particular, the downward shift in energy for the $\mathrm{Gr}$ states in $\mathrm{Gr} / 1 \mathrm{ML}-\mathrm{Co} / \mathrm{Ir}(111)$ with respect to $\mathrm{Gr} / \mathrm{Ir}(111)$ is well reproduced by calculations.

Interestingly, an energy shift of about $2 \mathrm{eV}$ of the occupied Gr $\pi$-bands at the $\bar{\Gamma}$ point has also been reported in (direct) photoemission upon 1ML-Co intercalation. ${ }^{19,62}$ However, both empty state dispersion and the good agreement with theoretical results prove that the Co intercalation effect cannot be simply modeled as a downshift of the Gr-bands, but rather needs to include hybridization effects, which are expected also for empty states of the Gr/1ML$\mathrm{Co} / \mathrm{Ir}(111)$ interface, close to the Fermi level. Unambiguous detection of such states by IPES is prevented by the presence of the Ir states dominating the spectra close to the Fermi level. However, an experimental signature has been already clearly observed in the broadening of the $\mathrm{C} \mathrm{K}$ edge ascribed to hybridized Co-C states, confirmed by the computed projected density of states shown in Fig. 2.

\section{CONCLUSIONS}

The intercalation of Co on $\mathrm{Gr} / \operatorname{Ir}(111)$ induces a significant modification of the $\mathrm{Gr}$ states at the interface, while Co deposition on top of $\mathrm{Gr} / \operatorname{Ir}(111)$ preserves the graphene electronic structure. Thanks to the negligible bulk solubility of Co atoms in the Ir substrate, Co sandwiched between Ir and Gr constitutes a single homogeneous layer influencing the $\mathrm{Gr}$ electronic structure. The downward shift of the Dirac cone is unveiled by AR-IPES and DFT calculations. A larger corrugation of Gr upon Co intercalation and the symmetry breaking of the $\pi / \sigma$ states hybridized with the metallic Co bands induce also a broadening of the NEXAFS features for $\mathrm{Gr} / 1 \mathrm{ML}-\mathrm{Co} / \mathrm{Ir}(111)$ when compared with $\mathrm{Gr} / \mathrm{Ir}(111)$, as confirmed by the evolution of the projected density of empty states deduced by DFT calculations.

A description of the changes occurring to the empty-state electronic structure during the various steps leading to the formation of Co-intercalated $\mathrm{Gr} / \operatorname{Ir}(111)$, starting from the bare $\operatorname{Ir}(111)$ substrate, is unraveled by AR-IPES. Thanks to its angular resolution, it provides complementary information on the spectroscopic features related to the Gr layer. In particular, the unoccupied $\pi^{*}$ states shift away from the Fermi level along the $\bar{\Gamma}-\overline{\mathrm{K}}$ direction. It is worth noting that the deposition of Co on top of the $\mathrm{Gr} / \operatorname{Ir}(111)$ structure does not modify the Dirac cone, consistent with the formation of Co clusters, ${ }^{32}$ while the Co-intercalation induces a shift of the Dirac cone and a symmetry breaking of the $\pi / \sigma$ states. The image states of $\operatorname{Ir}(111)$, more sensitive to the potential at the surface, are almost unperturbed when Gr covers the surface, even when the Co is deposited on the Gr cover, while they are depressed and shifted by the potential altered by the intercalated Co layer. Combined AR-IPES, NEXAFS, and DFT have proved to be particularly effective in determining in detail the empty electronic structure of interacting graphene systems, unveiling the clear transition from a nearly freestanding to a hybridized character of the graphene band structure.

\section{SUPPLEMENTARY MATERIAL}

See the supplementary material for the detailed description of the core-hole positions used to average the computed NEXAFS spectra, the similarity of the DOS for $\mathrm{Gr}$ and $\mathrm{Gr} / \mathrm{Ir}(111)$, and for $\mathrm{Gr} / \mathrm{Co}(0001)$ and $\mathrm{Gr} / 1 \mathrm{ML}-\mathrm{Co} / \mathrm{Ir}(111)$. AR-IPES spectra acquired on the $\operatorname{Ir}(111)$ substrate and a figure summarizing the major spectroscopic changes occurring during the various phases of Co intercalation are also included.

\section{ACKNOWLEDGMENTS}

We gratefully acknowledge stimulating discussions with Dario A. Leon. We also acknowledge Elettra Sincrotrone Trieste for providing access to its synchrotron radiation facilities and thank P. Lacovig for assistance in using beamline SuperESCA. This work was partially supported by the $\mathrm{MaX}$-MAterials design at the eXascale-Centre of Excellence, funded by the European Union program H2020-INFRAEDI-2018-1 (Grant No. 824143). Computational time on the Marconi-A2 machine at CINECA was provided by the Italian ISCRA program.

\section{DATA AVAILABILITY}

The data that support the findings of this study are available within the article and its supplementary material.

\section{REFERENCES}

${ }^{1}$ H. Vita, S. Böttcher, P. Leicht, K. Horn, A. Shick, and F. Máca, Phys. Rev. B 90, 165432 (2014).

${ }^{2}$ G. Avvisati, C. Cardoso, D. Varsano, A. Ferretti, P. Gargiani, and M. G. Betti, Nano Lett. 18, 2268 (2018).

${ }^{3}$ M. Bazarnik, J. Brede, R. Decker, and R. Wiesendanger, ACS Nano 7, 11341 (2013).

${ }^{4}$ G. Avvisati, P. Gargiani, P. Mondelli, F. Presel, A. Baraldi, and M. G. Betti, Phys. Rev. B 98, 115412 (2018).

${ }^{5}$ G. Avvisati, P. Mondelli, P. Gargiani, and M. G. Betti, Appl. Surf. Sci. 432, 2 (2018).

${ }^{6}$ G. Avvisati, P. Gargiani, D. Lizzit, M. Valvidares, P. Lacovig, C. Petrillo, F. Sacchetti, and M. G. Betti, Appl. Surf. Sci. 527, 146599 (2020).

${ }^{7}$ A. K. Geim and K. S. Novoselov, Nat. Mater. 6, 183 (2007).

${ }^{8}$ E. Sutter, P. Albrecht, F. E. Camino, and P. Sutter, Carbon 48, 4414 (2010).

${ }^{9}$ A. K. Geim, Science 324, 1530 (2009).

${ }^{10}$ L. Huang, Y. Pan, L. Pan, M. Gao, W. Xu, Y. Que, H. Zhou, Y. Wang, S. Du, and H.-J. Gao, Appl. Phys. Lett. 99, 163107 (2011).

${ }^{11}$ R. Decker, J. Brede, N. Atodiresei, V. Caciuc, S. Blügel, and R. Wiesendanger, Phys. Rev. B 87, 041403 (2013).

${ }^{12}$ I. Forbeaux, J.-M. Themlin, and J.-M. Debever, Phys. Rev. B 58, 16396 (1998).

${ }^{13}$ P. Sutter, J. T. Sadowski, and E. Sutter, Phys. Rev. B 80, 245411 (2009).

${ }^{14}$ M. Scardamaglia, G. Forte, S. Lizzit, A. Baraldi, P. Lacovig, R. Larciprete, C. Mariani, and M. G. Betti, J. Nanopart. Res. 13, 6013 (2011).

${ }^{15}$ I. Pletikosić, M. Kralj, P. Pervan, R. Brako, J. Coraux, A. T. N’Diaye, C. Busse, and T. Michely, Phys. Rev. Lett. 102, 056808 (2009).

${ }^{16}$ A. T. N'Diaye, M. Engler, C. Busse, D. Wall, N. Buckanie, F.-J. M. zu Heringdorf, R. van Gastel, B. Poelsema, and T. Michely, New J. Phys. 11, 039801 (2009).

${ }^{17}$ A. Dahal and M. Batzill, Nanoscale 6, 2548 (2014).

${ }^{18}$ D. Pacilè, P. Leicht, M. Papagno, P. M. Sheverdyaeva, P. Moras, C. Carbone, K. Krausert, L. Zielke, M. Fonin, Y. S. Dedkov, F. Mittendorfer, J. Doppler, A. Garhofer, and J. Redinger, Phys. Rev. B 87, 035420 (2013). 
${ }^{19}$ D. Pacilè, S. Lisi, I. Di Bernardo, M. Papagno, L. Ferrari, M. Pisarra, M. Caputo, S. K. Mahatha, P. M. Sheverdyaeva, P. Moras, P. Lacovig, S. Lizzit, A. Baraldi, M. G. Betti, and C. Carbone, Phys. Rev. B 90, 195446 (2014).

${ }^{20}$ L. Kong, C. Bjelkevig, S. Gaddam, M. Zhou, Y. H. Lee, G. H. Han, H. K. Jeong, N. Wu, Z. Zhang, J. Xiao, P. A. Dowben, and J. A. Kelber, J. Phys. Chem. C 114, 21618 (2010).

${ }^{21}$ M. S. Jagadeesh, A. Calloni, G. Bussetti, L. Duò, and F. Ciccacci, Phys. Status Solidi 255, 1700415 (2018).

${ }^{22}$ S. Bhardwaj, M. Kumar, C. C. Cudia, M. Pedio, and C. Cepek, Phys. Status Solidi 249, 2519 (2012).

${ }^{23}$ S. Tognolini, S. Achilli, L. Longetti, E. Fava, C. Mariani, M. I. Trioni, and S. Pagliara, Phys. Rev. Lett. 115, 046801 (2015).

${ }^{24}$ Y. Lin, Y. Li, J. T. Sadowski, W. Jin, J. I. Dadap, M. S. Hybertsen, and R. M. Osgood, Jr., Phys. Rev. B 97, 165413 (2018).

${ }^{25}$ S. Bose, V. M. Silkin, R. Ohmann, I. Brihuega, L. Vitali, C. H. Michaelis, P. Mallet, J. Y. Veuillen, M. A. Schneider, E. V. Chulkov, P. M. Echenique, and K. Kern, New J. Phys. 12, 023028 (2010).

${ }^{26}$ V. M. Silkin, J. Zhao, F. Guinea, E. V. Chulkov, P. M. Echenique, and H. Petek, Phys. Rev. B 80, 121408 (2009).

${ }^{27}$ N. Armbrust, J. Güdde, P. Jakob, and U. Höfer, Phys. Rev. Lett. 108, 056801 (2012).

${ }^{28}$ G. Berti, A. Calloni, A. Brambilla, G. Bussetti, L. Duò, and F. Ciccacci, Rev. Sci. Instrum. 85, 073901 (2014).

${ }^{29}$ A. Calloni, M. Cozzi, M. S. Jagadeesh, G. Bussetti, F. Ciccacci, and L. Duò, J. Phys.: Condens. Matter 30, 015001 (2018).

${ }^{30}$ I. Pletikosić, M. Kralj, D. Šokčević, R. Brako, P. Lazić, and P. Pervan, J. Phys.: Condens. Matter 22, 135006 (2010).

${ }^{31}$ G. Avvisati, S. Lisi, P. Gargiani, A. Della Pia, O. De Luca, D. Pacilè, C. Cardoso, D. Varsano, D. Prezzi, A. Ferretti, and M. G. Betti, J. Phys. Chem. C 121, 1639 (2017).

${ }^{32}$ J. Drnec, S. Vlaic, I. Carlomagno, C. J. Gonzalez, H. Isern, F. Carlà, R. Fiala, N. Rougemaille, J. Coraux, and R. Felici, Carbon 94, 554 (2015).

${ }^{33}$ I. Carlomagno, J. Drnec, A. M. Scaparro, S. Cicia, S. Vlaic, R. Felici, and C. Meneghini, J. Appl. Phys. 120, 195302 (2016).

${ }^{34}$ J. E. Bickel, F. Meier, J. Brede, A. Kubetzka, K. von Bergmann, and R. Wiesendanger, Phys. Rev. B 84, 054454 (2011).

${ }^{35}$ D. T. Pierce, R. J. Celotta, G.-C. Wang, W. N. Unertl, A. Galejs, C. E. Kuyatt, and S. R. Mielczarek, Rev. Sci. Instrum. 51, 478 (1980).

${ }^{36}$ F. Ciccacci and G. Chiaia, J. Vac. Sci. Technol., A 9, 2991 (1991).

${ }^{37}$ F. Ciccacci, E. Vescovo, G. Chiaia, S. De Rossi, and M. Tosca, Rev. Sci. Instrum. 63, 3333 (1992).

${ }^{38}$ M. Finazzi, A. Bastianon, G. Chiaia, and F. Ciccacci, Meas. Sci. Technol. 4, 234 (1993).

${ }^{39}$ F. Ciccacci, S. De Rossi, A. Taglia, and S. Crampin, J. Phys.: Condens. Matter 6 , 7227 (1994)

${ }^{40}$ P. Giannozzi, S. Baroni, N. Bonini, M. Calandra, R. Car, C. Cavazzoni, D. Ceresoli, G. L. Chiarotti, M. Cococcioni, I. Dabo et al., J. Phys.: Condens. Matter 21, 395502 (2009).

${ }^{41}$ P. Giannozzi, O. Andreussi, T. Brumme, O. Bunau, M. Buongiorno Nardelli, M. Calandra, R. Car, C. Cavazzoni, D. Ceresoli, M. Cococcioni, N. Colonna, I. Carnimeo, A. Dal Corso, S. de Gironcoli, P. Delugas, R. A. DiStasio, Jr.,
A. Ferretti, A. Floris, G. Fratesi, G. Fugallo, R. Gebauer, U. Gerstmann, F. Giustino, T. Gorni, J. Jia, M. Kawamura, H.-Y. Ko, A. Kokalj, E. Küçükbenli, M. Lazzeri, M. Marsili, N. Marzari, F. Mauri, N. L. Nguyen, H.-V. Nguyen, A. O. de-la Roza, L. Paulatto, S. Poncé, D. Rocca, R. Sabatini, B. Santra, M. Schlipf, A. P. Seitsonen, A. Smogunov, I. Timrov, T. Thonhauser, P. Umari, N. Vast, X. Wu, and S. Baroni, J. Phys.: Condens. Matter 29, 465901 (2017).

${ }^{42}$ J. P. Perdew and A. Zunger, Phys. Rev. B 23, 5048 (1981).

${ }^{43}$ O. Bunău and M. Calandra, Phys. Rev. B 87, 205105 (2013).

${ }^{44}$ C. Gougoussis, M. Calandra, A. P. Seitsonen, and F. Mauri, Phys. Rev. B 80, 075102 (2009).

${ }^{45}$ M. Taillefumier, D. Cabaret, A.-M. Flank, and F. Mauri, Phys. Rev. B 66, 195107 (2002).

${ }^{46}$ L. Triguero, L. G. M. Pettersson, and H. Ågren, Phys. Rev. B 58, 8097 (1998).

${ }^{47}$ B. Hetényi, F. De Angelis, P. Giannozzi, and R. Car, J. Chem. Phys. 120, 8632 (2004).

${ }^{48}$ M. Cavalleri, M. Odelius, D. Nordlund, A. Nilsson, and L. G. M. Pettersson, Phys. Chem. Chem. Phys. 7, 2854 (2005).

${ }^{49}$ G. Fratesi, S. Achilli, N. Manini, G. Onida, A. Baby, A. Ravikumar, A. Ugolotti, G. P. Brivio, A. Milani, and C. S. Casari, Materials 11, 2556 (2018).

${ }^{50}$ A. T. N'Diaye, J. Coraux, T. Plasa, C. Busse, and T. Michely, New J. Phys. 10, 043033 (2008).

${ }^{51}$ D. A. Duncan, N. Atodiresei, S. Lisi, P. J. Blowey, V. Caciuc, J. Lawrence, T.L. Lee, M. G. Betti, P. K. Thakur, A. Della Pia, S. Blügel, G. Costantini, and D. P. Woodruff, Phys. Rev. Mater. 3, 124001 (2019).

${ }^{52} \mathrm{~K}$. Suenaga and M. Koshino, Nature 468, 1088 (2010).

${ }^{53}$ D. Pacilè, M. Papagno, A. F. Rodríguez, M. Grioni, L. Papagno, Ç. Ö. Girit, J. C. Meyer, G. E. Begtrup, and A. Zettl, Phys. Rev. Lett. 101, 066806 (2008).

${ }^{54}$ S. Entani, S. Ikeda, M. Kiguchi, K. Saiki, G. Yoshikawa, I. Nakai, H. Kondoh, and T. Ohta, Appl. Phys. Lett. 88, 153126 (2006).

${ }^{55}$ M. Scardamaglia, S. Lisi, S. Lizzit, A. Baraldi, R. Larciprete, C. Mariani, and M. G. Betti, J. Phys. Chem. C 117, 3019 (2013).

${ }^{56}$ M. Weser, Y. Rehder, K. Horn, M. Sicot, M. Fonin, A. B. Preobrajenski, E. N. Voloshina, E. Goering, and Y. S. Dedkov, Appl. Phys. Lett. 96, 012504 (2010).

${ }^{57}$ D. Niesner, T. Fauster, J. I. Dadap, N. Zaki, K. R. Knox, P.-C. Yeh, R. Bhandari, R. M. Osgood, M. Petrović, and M. Kralj, Phys. Rev. B 85, 081402 (2012).

${ }^{58}$ D. Straub and F. J. Himpsel, Phys. Rev. B 33, 2256 (1986).

${ }^{59}$ S. L. Hulbert, P. D. Johnson, N. G. Stoffel, W. A. Royer, and N. V. Smith, Phys. Rev. B 31, 6815 (1985).

${ }^{60}$ A. Varykhalov, D. Marchenko, M. R. Scholz, E. D. L. Rienks, T. K. Kim, G. Bihlmayer, J. Sánchez-Barriga, and O. Rader, Phys. Rev. Lett. 108, 066804 (2012).

${ }^{61}$ I. Schäfer, M. Schlüter, and M. Skibowski, Phys. Rev. B 35, 7663 (1987).

${ }^{62}$ S. Vlaic, A. Kimouche, J. Coraux, B. Santos, A. Locatelli, and N. Rougemaille, Appl. Phys. Lett. 104, 101602 (2014).

${ }^{63}$ See also Fig. 57 of the supplementary material summarizing the major spectroscopic changes occurring during the various phases of Co intercalation.

${ }^{64}$ V. Popescu and A. Zunger, Phys. Rev. Lett. 104, 236403 (2010).

${ }^{65}$ P. V. C. Medeiros, S. Stafström, and J. Björk, Phys. Rev. B 89, 041407 (2014).

${ }^{66}$ P. V. C. Medeiros, S. S. Tsirkin, S. Stafström, and J. Björk, Phys. Rev. B 91, 041116 (2015). 